\title{
Below Elbow Upper Limb Prosthetic for Amputees and Paralyzed Patients
}

\author{
Debika Khanra \\ Department of Biomedical Engineering \\ VIT University \\ Vellore, India
}

\author{
Dr. S. Sudesh \\ Department of Biomedical Engineering \\ VIT University \\ Vellore, India
}

\begin{abstract}
An artificial limb is a type of prosthesis that replaces a missing extremity, such as arms or legs. The type of artificial limb required is determined by the availability of the left over stump after amputation. The main problem faced by the patients after amputation or paralysis is muscle atrophy caused due to nonfunctionality of the stump. The limited functionality leads to very limited flow of oxygenated blood to the stump causing muscle wasting which is a very big medical problem. Moreover after amputation or paralysis the patients become dependent on others for their basic functions or jobs which lead to serious psychological problems. This paper deals with the design of an upper limb below elbow prosthetic which can be used by both amputees and paralyzed patients to bring about simple hand movements independently by the patient. The electro-mechanical prosthetic arm will be a hollow shell structure supported by a neck and waist harness for the patient's support.
\end{abstract}

\section{General Terms}

Design of Prosthetic, Prototype development

\section{Keywords}

Artificial limbs, Upper limb Prosthetics, Amputee Prosthetics, Electro-mechanical Arm

\section{INTRODUCTION}

Prosthesis is a device that is intended to substitute, as much as achievable, the function or appearance of a missing limb or body part. The type of artificial limb is determined largely by the extent of an amputation or loss of the missing extremity. As the biological system of the hands is very highly suited for their functioning, so their man-made replacement should also involve considerable levels of complexity in both design and construction. Thus for designing prosthetic limbs, it is necessary for the designer to closely examine natural limb systems and how various functions are biologically accomplished. Most importantly identify the essential performance features which have to be replicated. This will help in the precise and more accurate combination of the man-made components for at least partially recovering the lost human hand functionality. The main aim of the artificial limb designers is to fabricate a product that is smooth, friction free, less power consuming for its best use by the amputees for their benefit and service. In the $19^{\text {th }}$ century, artificial limbs became more widespread due to the large number of amputees from wars such as the Napoleonic Wars in Europe and American Civil War and then the World Wars. In recent years, a great deal of emphasis has been placed on developing artificial limbs that look and function more like actual human limbs. Advances in biomedical understanding, through combined work of the doctors and engineers, and use of computer aided designs, signal processing and manufacturing have contributed in the progress in this field. But the artificial limbs that are presently available in the market have many drawbacks. Firstly, the functional artificial limbs- 'myo-electric arms' make use of EMG signals which are not stable source of signal as they tend to vary from time to time and also according to the muscle activity. They are generally expensive because of the use of complex electronics which common people are not able to afford. Secondly, the lowcost prosthetic arms have very restricted functionality. Then there is also the problem of maintenance of these myo-electric arms because of the electrical components used. Also it is a tough job for the layman or common people to master the high level technology used for the functioning of the myo-electric arm. 


\section{CHALLENGES FACED BY AMPUTEES} AND PARALYZED PATIENT

Upper limb amputees account for approximately $26.5 \%$ of the world's population. And the most common cause apart from congenital disease or disability is trauma, either in wars, occupational accidents or traffic accidents. The main problem faced by the patients after amputation or paralysis is the nonfunctionality of the leftover stump. Due to this reason there is very limited flow of oxygenated blood to the muscle wasting which is a very big medical problem. The patients become dependent on others for their basic functions or jobs which lead to serious psychological problems. Post-amputation an amputee has to survive and face major physical, social, and emotional challenges and adjustments. Upper limb amputation results in severe limitations both at functional and aesthetic levels and in many cases it can be distressing as it represents a socio-economic catastrophe for the patient. The reintegration of the patient in the social and work environment is influenced by several conditions like personal and surrounding environmental factors. The personal factor may include physical, psychological and cultural circumstance of the patient and environmental factor is related to the social, family and working condition. The adaptation level varies widely with varying factors but little is known about which factors exactly contribute to successful adjustment.

\section{MATERIALS AND METHOD}

In this paper an innovative and simple design has been discussed for an upper limb below elbow prosthetic which can be used by both amputees and paralyzed patients. The arm has a shell or brace like structure which can be worn by the patient and strapped on to the left over stump. It will provide support to the limp arm of the paralyzed patient and for the amputees it will act as an artificial hand. The waist band will have an on/ off switch to start and stop the hand movement. For the amputee patients the length of the arm below the elbow can be customized according to his/ her needs. The whole frame will be supported by a neck and waist harness for the comfort of the patient. The main purpose of this hand is to give simple movements to the fingers like flexion, extension and grabbing of objects. This will give functionality to the patient's upper limb and assist him to accomplish simple day to day activities independently.

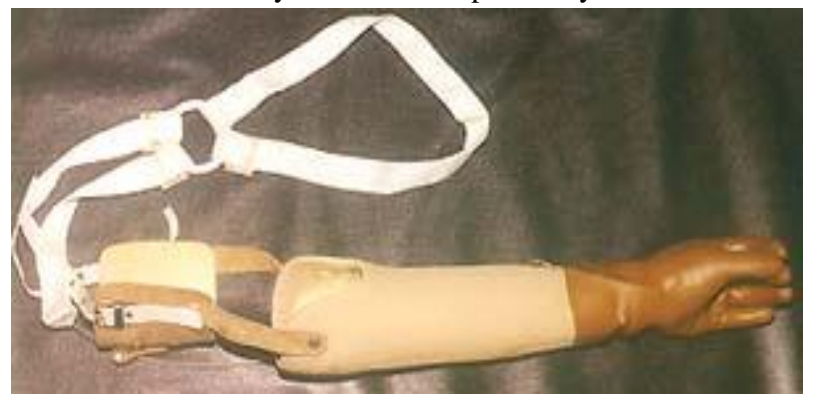

Fig 1 - Shell of the prosthesis

\subsection{Block Diagram Description}

The block diagram of the below elbow upper limb prosthetic is given in Fig 2. It shows the step by step design of the prosthetic in a simple manner.

The prosthetic arm shell is made up of plastic and is covered by a cosmetic glove made of polyurethane to enhance the aesthetic appearance of the hand. The prosthesis is attached to the stump and has a shoulder and neck harness for giving support to the patient and reducing the burden of prosthetic. An on/off switch is provided on the waist harness to control the start and stop of the hand movement. The switch is connected to the micro-controller to control the motor movement. The micro-controller is programmed to control the motor movement. The shaft of the motor is attached to the external spur gear box to obtain high torque value. The gearing unit is connected to the springs. Metal springs of medium spring constant $(\mathrm{K})$ value has been used. The springs are attached on the upper part of the fingers. In this design only three fingers have been made functional, the thumb, index and the middle finger. One motor is used to operate the thumb spring and the other motor to operate the index and middle finger springs, these later two being inter-connected. When the motor rotates, the gear attached to it also rotates and it pulls the spring attached to it outwards. Thus the fingers move outwards and the hand opens. And when the motor is stopped the spring moves back to its original position that is inwards. Thus the voluntary opening action of thumb, index finger and middle finger operate against spiral spring force. Any object which comes in between can be grabbed easily using the prosthetic arm.

\subsection{Circuit Diagram Explanation}

The circuitry for the motor control using micro-controller is given in Fig.3. AT89C51 micro-controller and L23D motor driver is used. $12 \mathrm{~V}$ D.C planetary geared carbon brush motor of $60 \mathrm{rpm}$ has been used. It has cylindrical body with a diameter of 1.77 inch, output power of $0.5 \mathrm{HP}$, continuous current of 4.5-5.5 amps and torque value of $0.6 \mathrm{ln}$-lbs. The battery used for power supply is a general Li-ion mobile battery of $3.7 \mathrm{~V}$ and $1020 \mathrm{mAH}$ storage capacity. The voltage is stepped up using a step up IC to obtain $12 \mathrm{~V}$ supply for the motor and IC 7805 is used to obtain $5 \mathrm{~V}$ for the micro-controller functioning. The battery runs for more than two hours of continuous usage but mostly depends on the load the hand is lifting; it can be recharged using an universal charger.

AT89C51 micro-controller is controlled by an external on/off switch and is programmed to control the motor. The logic behind the program is: port P1 is set as the 'input port' and port P2 is set as the 'output port'. First the accumulator cleared and assigned $00 \mathrm{H}$ value so that the motor is at rest in normal condition. When the switch is pressed ' $\mathrm{ON}$ ' the accumulator value is $01 \mathrm{H}$ and the motor rotates and this continues in a loop till the switch is pressed 'ON'. When the switched 'OFF' the accumulator value becomes $00 \mathrm{H}$ and the motor stops rotating. 


\section{AMPUTEE ACCEPTANCE OF PRODUCT}

The product to be used needs to satisfy some indispensable criteria to be accepted by the patients. It varies from the availability of the product in the local area, the cost of the prosthetic, technicians to educate the user how to use the product, personnel available for the maintenance and support, the longevity of the prosthetic, the assistance it provides and ease of use, etc. Some of the factors has been discussed in details here.

4.1 Availability: A prosthetic arm on average is used for $6000 \mathrm{hrs} / y e a r$. Since it is used for doing the daily chores with minimum maintenance and support, the modular components must be rugged to reduce unintentional damage, self damage and must be reliable for long term usage. In case of failure, the maintenance must be simple and fast to increase the availability of the prosthetic to the amputee.

4.2 Comfort and Feel: The quality of the socket and harness, the total weight of the system determines the comfort and 'feel' of the prosthesis for the user. By the use of proper control systems and hardware, the prosthetic should function as a part of the amputee's body and not robot like stiff appendage.

4.3 Performance: The performance of the prosthetic is a result of the control system and the hardware. 4.3.1 The Control System extracts the mechanical/electrical 'signals' from the amputee to perform signal processing, combine them to take decisions and supervise the system function. In short the controller has to determine what task the amputee would perform and asks the hardware to execute it. The 'sites' for the acquisition should be reliable and minimum with non-invasive monitoring elements. The control system must be natural, reasonable to learn, reliable and stable over time, flexibility in variety of amputee application and its operation should be graceful.

4.3.2 The Hardware has many design constraints which determine the performance requirements. They can be broadly classified as: [i] Structures- the structural components should be have good strength, minimum weight, water and dust resistant, have aesthetic look and immune to external forces. [ii] Actuation Systems- the externally powered actuators should be able to provide torques sufficient enough to handle loads up to $2 \mathrm{~kg}$. The actuation systems should be disengagable to prevent damage to transmission systems during certain movements. It is also important to have low mechanical output impedance of the actuators for degrees of freedom to achieve graceful, freeswinging operation. [iii] Electronic Systems- the electronic systems used in the complex prosthetics are also highly complex implementing control and power modulation functions. The electronic hardware should have minimum volume and weight, good computational speed versus power consumption constraints and precise design of all the wiring and connector systems.

4.4 Appearance: The appearance of the prosthetic is an important parameter for the amputee for his social acceptance. It is a result of the combination of cosmetic, motion and acoustic cues. It should also be provided with color, texture and shape which replicate the natural arm keeping in mind that it does not interfere with the performance. Graceful movements enhance the beauty and visual anonymity of the limb and also help the prosthetic to operate in sync.

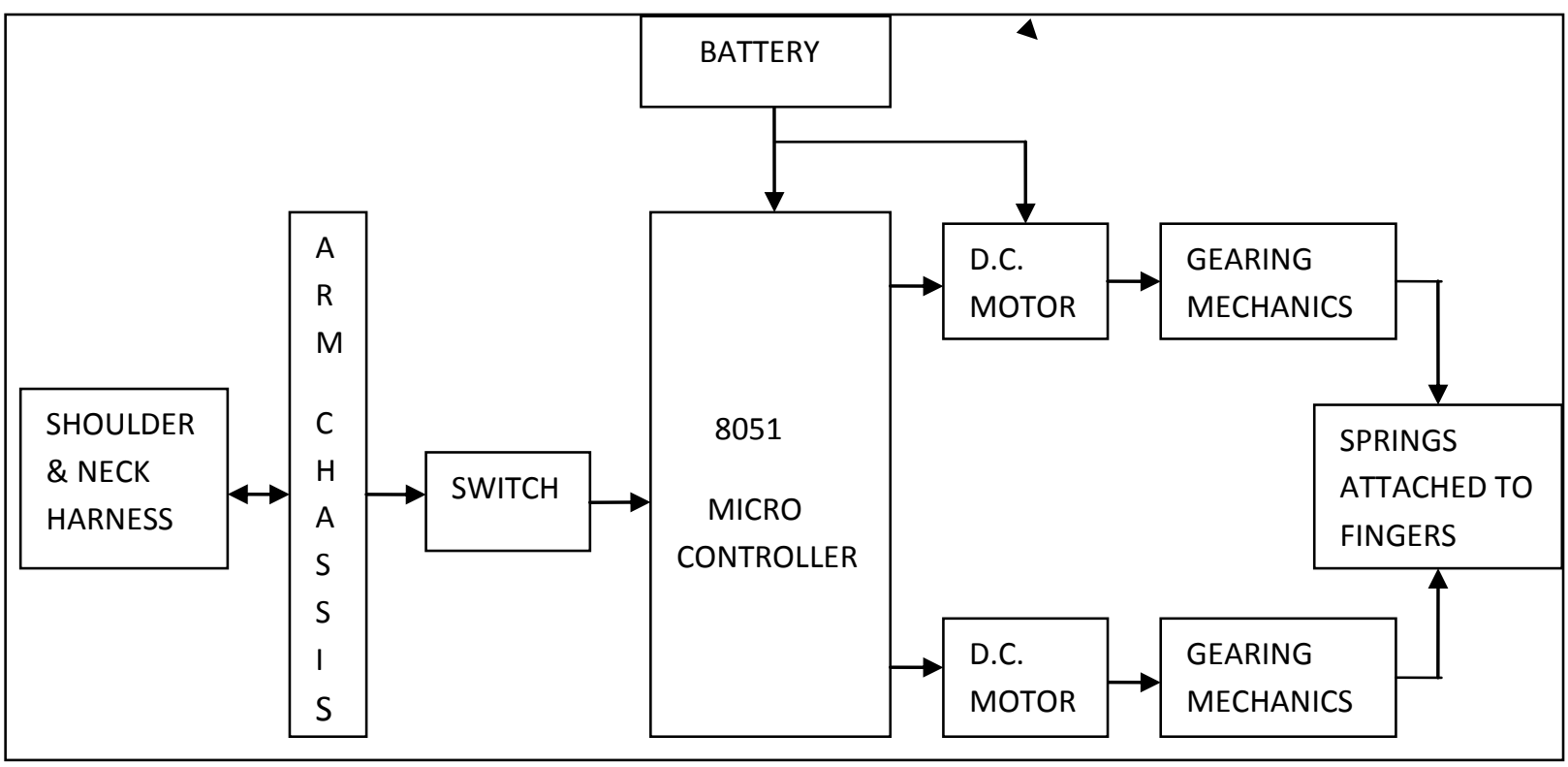

Fig 2 - Block diagram 


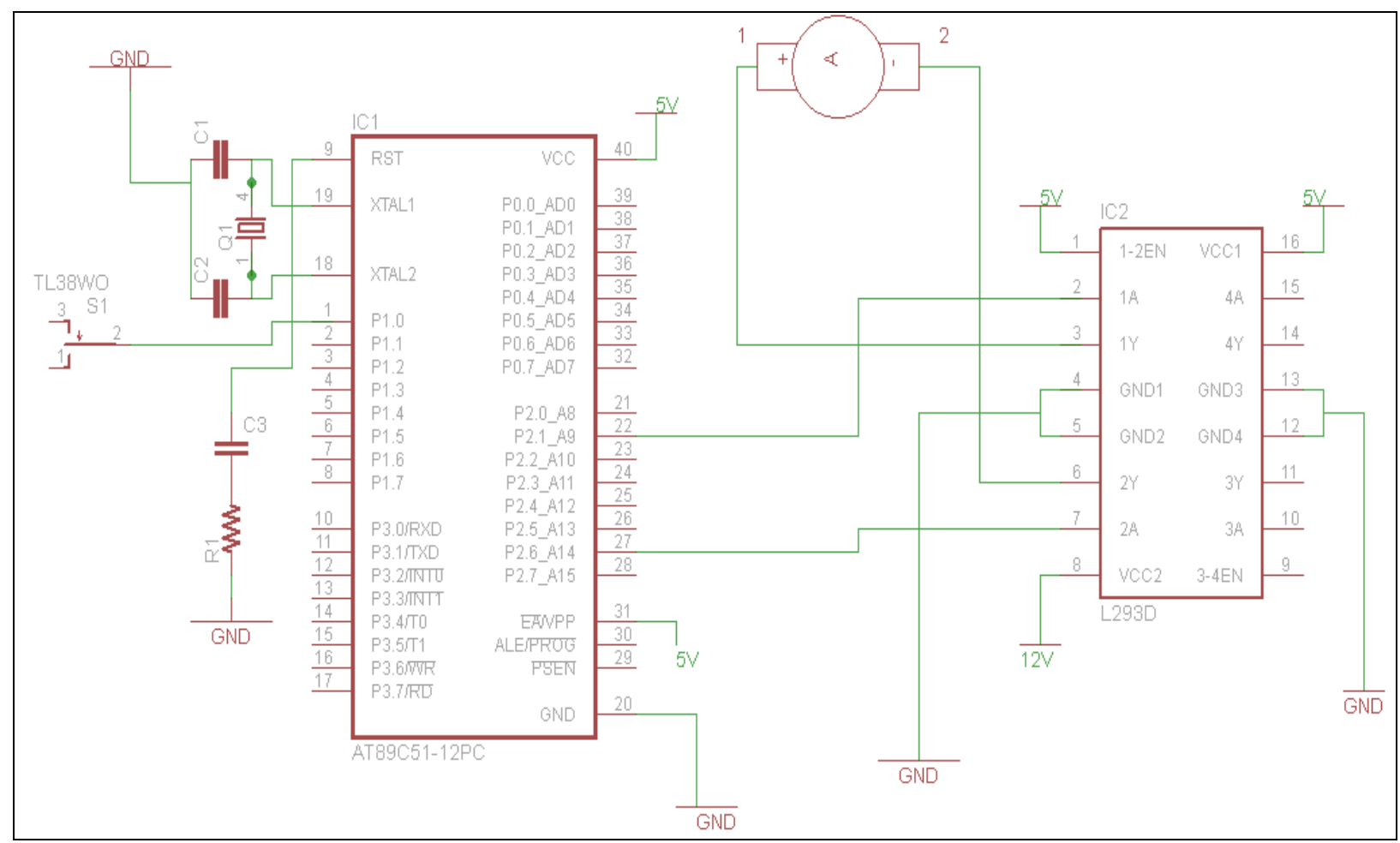

Fig 3 - Circuit diagram for switch controlled DC motor

\section{RESULT AND DISCUSSION}

It has been found out through extensive surveys with patients in rehabilitation clinics and hospitals, that after the initial shock of amputation wears off, mostly within a year or two, the amputees stop wearing their prostheses. The discomfort caused by the arm socket, where the prosthesis is connected to the body, is one of the chief reasons why the amputees stop wearing their prosthetics. By the traditional connection method the prosthetic is designed to create the greatest possible surface area for connecting the stump to the prosthetic; basically the amputee's stump is stuffed into the prosthesis. But the strain of daily use often results in a sweaty, slippery connection that makes proper use of the prosthesis almost impossible.

The below elbow upper limb design discussed in this is simple yet helps the patient to perform some basic functions with their arm like grabbing of objects. The most important advantage is that it can be used both by amputees and paralyzed patients as it has a shell kind structure. It also brings back muscle movement to the paralyzed patient's hand and reduces muscle atrophy.

\section{ACKNOWLEDGMENT}

I would like to acknowledge the contributions made by my guides Dr.S.Sudesh, divisional Leader \& Assistant Professor; Biomedical Division, SBST; VIT University and Col.K.Radhakrishnan, Managing Director, Worth Trust; Vellore and my friend Sriram K.V. for their constant help and guidance in completion of this paper.

\section{REFERENCES}

[1] Nei A. Andre, Geovany A. Borges, Francisco A. de O. (2007). A New Biomechanical Hand Prosthesis controlled by surface electromyographic signals. $29^{\text {th }}$ Annual International Conference of the IEEE EMBS Cite Internationae, Lyon, Nascimento, Alexandre R.S. Romariz and Andson F. da Rocha 624-632.

[2] Saqib Ahmed, Imran Khan, Saif Ullah, J.Iqbal, Z.Riaz, "Design and Fabrication of an Efficient Automated Prosthetic Above Elbow Joint with Body Powered Gripper." Department of Mechatronics Engineering, National University of Science and Technology (NUST) 
Rawalpindi, Pakistan.

[3] C.P.Mason,(1972). "Design of a powered prosthetic arm system for the above-elbow amputee" Bull. Prosth. Res.,Fall 1972.

[4] S. W. Alderson, "The electric arm," in Human Limbs and Their Substitutes, Klopsteg and Wilson, Ed. New York: Hafner, 1968, pp. 359-410 (reprint of McGraw-Hill, 1954).

[5] Stephen c. Jacobsen, David f. Knutti, Richard t. Johnson, and Harold Sears; "Development of the Utah Artificial Arm", IEEE Transactions on Biomedical Engineering, vol. BME-29, no. 4, Apri 1982
[6] Jacobsen S.C., Wood J.E. and D.F. Knutti (1984). The UTAH/ MIT dexterous hand: work in progress. The International Journal of Robotics Research,vol.4 pp.25-50

[7] Massa B., S. Roccella, M.C. Carrozza and P.Dario (2002). Design and Development of an under actuated Prosthetic Hand, $26^{\text {th }}$ IEEE International Conference on Robotics \& Automation, pp. 3374-3379.

[8] Carrozza M.C., G. Cappiello, L.Beccai, F. Zaccone, S. Micera, P.Dario (2004). Design Methods for Innovative Hand Prostheses. 24 ${ }^{\text {th }}$ Annual Conference of the IEEE EMBS, San Fransisco, pp.352-361. 